\title{
IMAGE NETWORK GENERATION OF UNCALIBRATED UAV IMAGES WITH LOW- COST GPS DATA
}

\author{
Shan Huang a , Zuxun Zhang a , Jianan $\mathrm{He}^{\mathrm{a}}$, Tao $\mathrm{Ke}^{\mathrm{a}, *}$ \\ a School of Remote Sensing and Information Engineering, Wuhan University, No.129 Luoyu Road, Wuhan, China - \\ huangsh@whu.edu.cn, zhangzx@cae.cn, (hjn2010, ketao)@whu.edu.cn
}

Commission III, WG III/1

KEY WORDS: UAV images, uncalibrated camera, Image Network Generation, GPS, Space Coordinate Transformation, Selfcalibration, Bundle adjustment

\begin{abstract}
:
The use of unmanned air vehicle (UAV) images acquired by a non-metric digital camera to establish an image network is difficult in cases without accurate camera model parameters. Although an image network can be generated by continuously calculating camera model parameters during data processing as an incremental structure from motion ( $\mathrm{SfM}$ ) methods, the process is time consuming. In this study, low-cost global position system (GPS) information is employed in image network generation to decrease computational expenses. Each image is considered as reference, and its neighbor images are determined based on GPS coordinates during processing. The reference image and its neighbor images constitute an image group, which is used to generate a free network through image matching and relative orientation. Data are then transformed from the free network coordinate system of each group into the GPS coordinate system by using the GPS coordinates of each image. After the exterior elements of each image are determined in the GPS coordinate system, the initial image network is established. Finally, self-calibration bundle adjustment constrained by GPS coordinates is conducted to refine the image network. The proposed method is validated on three fields. Results confirm that the method can achieve good image network when accurate camera model parameters are unavailable.
\end{abstract}

\section{INTRODUCTION}

Unmanned air vehicles (UAV) are commonly used for terrain measurements because of their low costs, mobilization, flexibility, and high spatial resolution (Gonçalves and Henriques, 2015; Hui et al., 1998; Uysal et al., 2015). Most UAV photogrammetry systems require a global position system (GPS) receiver and an inertial measurement unit to provide position and orientation information when photographing. However, some UAV photogrammetry systems only employ low-cost GPS receivers to decrease device weight and cost but rarely use the inertial measurement unit because it is expensive Consequently, these systems can only obtain the location of images with low precision. With the wide use of UAV images, some operators who control the machine to capture images do not have sufficient experience (Luhmann, 2010). For inexperienced users, the requirements for collecting suitable imagery for "conventional" photogrammetry are difficult to accomplish. As such, structure from motion (SfM) in computer vision is applied to reconstruct a 3D model by using irregular image data (Agarwal et al., 2011; Jiang et al., 2013; Schaffalitzky and Zisserman, 2002; Strecha et al., 2008). SfM is driven by enabling automated model reconstruction from imagery with few limitations in types of digital cameras and viewing angles (Uysal et al., 2015; Westoby et al., 2012).With the advantages of the modern digital camera technology, nonmetric cameras, such as digital single-lens reflex cameras (DSLRs), are widely used, particularly on the platform of UAVs However, non-metric cameras have significant radial distortions, and camera parameters are unstable. Moreover, non-metric digital cameras applied for measurements may not be calibrated.
As such, on-line calibration approach is adopted to refine image processing results. However, without accurate camera parameters, image network generation remains a challenge in reconstructing 3D models because of severe image distortion. Although all match correspondences are correct, radial distortion may lead to ambiguities when recovering motion and structure (Wu, 2014). Our previous research on processing UAV images without accurate camera parameters indicate that image position is reconstructed farther from the actual positions with increasing strip length, despite that some images are located lower than the ground points. Thus, coarse estimation for image network is significant for the final self-calibration bundle adjustment, when only approximate camera parameters can be extracted from image files without distortion information. Moreover, continuous estimation of camera model parameters when establishing image network with incremental SfM method is time consuming (Jiang et al., 2013; Wilson and Snavely, 2014).

Thus, an effective image network generation method without accurate camera intrinsic parameters must be developed. This process is a key technique to determine whether the final bundle adjustment converges or not. While, images are successively acquired by UAVs, and the locations of the images are obtained from GPS receivers, regardless if they exhibit high precision or not. As such, establishing a good image network is possible even without intrinsic parameters.

In this paper, we focus on generating effective coarse image networks without accurate intrinsic parameters by using lowprecision GPS coordinates of images. Self-calibration bundle

\footnotetext{
* Corresponding author
} 
adjustment with GPS coordinates as constraints was employed to obtain accurate results. We propose a method that employs GPS information to help align UAV images, instead of using GPS information for direct geo-reference only. First, we convert the GPS coordinates of the WGS-84 Earth Centered Earth Fixed coordinate system into the Euclidean coordinate system through Gauss Projection. Each image is considered as the reference, and its neighbor images are determined according to the GPS coordinates; these images are grouped together. Second, image matching and relative orientation between image pairs are implemented with the reference image as the left image and its neighbor images as the right image to generate a free network of the group. The origin of one group of free network is considered the perspective center of the reference image. Third, the position and orientation vectors of the perspective center are transformed from the relative coordinate system into the GPS coordinate system. As one image may contain several sets of exterior elements, which are computed from different groups, and only one set of exterior elements of each image is needed for bundle adjustment, the weight least square estimation algorithm is adopted to composite the data. Afterward, initial image network is generated. Finally, self-calibration bundle adjustment with GPS coordinates as constraints is performed to refine the image network. Evidently, the proposed method can be easily implemented and parallelized.

As UAV images are used in geographic survey, the focal length of the camera is fixed to maintain the precision of the measurement. Camera parameters are constant during the short time of data acquisition (Huang et al., 2015). Under this condition, we performed experiments on three fields to validate the proposed method for generating an image network. Results confirm that the method can achieve satisfactory performance when processing images with inaccurate camera intrinsic parameters.

The rest of this paper is organized as follows. The theories of the proposed image network generation are shown in Section 2. The performance of the proposed method is evaluated using real datasets in Section 3. The conclusion and recommendations are presented in Section 4.

\section{METHODOLOGY}

\begin{tabular}{|c|}
\hline GPS coordinates transformation \\
\hline Division of images into groups \\
\hline \begin{tabular}{|} 
Free network generation \\
(group images)
\end{tabular} \\
\hline \begin{tabular}{|} 
Space coordinates transformation \\
(from free network coordinate system to GPS coordinate system)
\end{tabular} \\
\hline Weighted least square estimation \\
\hline $\begin{array}{c}\text { Self-calibration bundle adjustment with } \\
\text { GPS coordinates }\end{array}$ \\
\hline
\end{tabular}

Figure 1. Flow chart of image network generation.

\subsection{Division of images into groups}

The coordinates are transformed into the Euclidean coordinate system through Gauss Projection before image network generation because the original GPS coordinates are observed in the WGS-84 coordinate system when output from the GPS receiver. All GPS coordinates referred below are in the Euclidean coordinate system.

Each image, which is set as the reference, and its neighbor images are grouped together. For simplification, neighbor images are determined according to their distance from the reference; the distance is computed using GPS coordinates. If the distance between an image and the reference image is lower than the distance threshold, the image is identified as the neighbor image of the reference. The distance threshold is set as three times of the average distance between image pairs, which are composed of adjacent images aligned according to acquisition time. The distance threshold is calculated using Equation (1).

$$
L=3 \cdot \frac{\sum_{i=1}^{N-1} \sqrt{\left(X_{G P S}^{i}-X_{G P S}^{i+1}\right)^{2}+\left(Y_{G P S}^{i}-Y_{G P S}^{i+1}\right)^{2}+\left(Z_{G P S}^{i}-Z_{G P S}^{i+1}\right)^{2}}}{N-1}
$$

where $\quad X_{G P S}^{i}, Y_{G P S}^{i}, Z_{G P S}^{i}=$ GPS coordinates of perspective center of image $i$

$N=$ total number of images

$L=$ distance threshold

\subsection{Generation of a free network for groups}

After each group is determined, the relative position and orientation of neighbor images should be recovered. Each neighbor image in a group should match to the reference image, and relative orientation should be performed. Afterward, each group can be used to establish a free network in its own coordinate system. Considering the topographic relief and the flight altitude using UAVs, we choose the scale-invariant feature transform (SIFT) match (Lowe, 2004) because this algorithm can be applied in many cases. However, mismatching is unavoidable in image matching methods; as such, we apply the random sample consensus algorithm (Fischler and Bolles, 1981) to estimate parameters of quadric polynomial model and discard outliers in the SIFT point matches. A five-point relative orientation algorithm (Stewenius et al., 2006) based on essential matrix is also employed to implement relative orientation. Although accurate camera intrinsic parameters are needed for five-point relative orientation algorithm, we can only utilize inaccurate camera intrinsic parameters obtained from image files. However, in our experience, when the residual threshold of the five-point relative orientation used for identifying outliers is relaxed, the method can maintain its satisfactory performance in recovering the relative attitude between image pairs in most cases.. The overlap between the reference and its neighbor images may be less than $50 \%$, particularly when two images are collected from different stripes. The absolute value of the relative position in $\mathrm{Z}$ direction should not be higher than the values in $\mathrm{X}$ and $\mathrm{Y}$ directions at the same time; this constraint is applied when executing relative orientation. Then, correct relative orientation can be obtained between image pairs in most cases. In the next step, error detection will be performed.

After the relative orientation procedure, the free network of one group is established. 


\subsection{Generation of image network}

In this step, we use GPS coordinates to transform all group free networks into one to generate an initial image network for bundle adjustment. We refine this image network by selfcalibration bundle adjustment with GPS coordinates.

The perspective center of the reference image is the origin of the free network of the group, and the coordinates of this image are known in the GPS coordinate system; thus, we can immediately obtained the translation between the two coordinate systems, and the values are the same as the GPS coordinates of the reference image. Therefore, we only need to work out rotation angles and scales between each free network coordinate system and the GPS coordinate system. For simplification, the value of the scale is determined using the ratio of distances between the reference and neighbor image in the two coordinate systems. The computed coordinates in the free network may be incorrect, but the GPS coordinates of each neighbor image are known. Hence, we decide that the scale of each neighbor image varies when normalizing coordinates from the coordinate system of the free network to the GPS coordinate system. Thus, mutual influence can be avoided. The scale is calculated as Equation (2).

$$
s_{i j}=\sqrt{\frac{\left(X_{G P S}^{i j}-X_{G P S}^{i}\right)^{2}+\left(Y_{G P S}^{i j}-Y_{G P S}^{i}\right)^{2}+\left(Z_{G P S}^{i j}-Z_{G P S}^{i}\right)^{2}}{\left(X_{r e l}^{i j}-X_{r e l}^{i}\right)^{2}+\left(Y_{r e l}^{i j}-Y_{r e l}^{i}\right)^{2}+\left(Z_{r e l}^{i j}-Z_{r e l}^{i}\right)^{2}}}
$$

where $X_{G P S}^{i}, Y_{G P S}^{i}, Z_{G P S}^{i}=$ GPS coordinates of the reference image in group $i$

$X_{G P S}^{i j}, Y_{G P S}^{i j}, Z_{G P S}^{i j}=$ GPS coordinates of neighbor image $j$ in group $i$

$X_{\text {rel }}^{i}, Y_{\text {rel }}^{i}, Z_{\text {rel }}^{i}=$ relative coordinates of the reference image in the free network coordinate system of group $i$, and the value is 0 .

$X_{r e l}^{i j}, Y_{r e l}^{i j}, Z_{r e l}^{i j}=$ relative coordinates of neighbor image $j$ in the free network coordinate system of group $i$

$s_{i j}=$ the scale of neighbor image $j$ in group $i$

After unifying the free network of each group into the same scale of the GPS coordinate system, the approximate rotation angles between the free network coordinate system and the object space coordinate system are computed according to the space projector algorithm (Slama et al., 1980; Wang, 2007). A set of transformation angles can be determined using the coordinates of an image pair composed of the reference and neighbor image through Equation (3).

As coordinates of each neighbor image in a group can be used to solve a set of rotation angles, we need to composite the values and determine the appropriate rotation angles between the two coordinate systems. As such, we rank the angles and select the median one as the initial value. To detect error and improve precision, we adopt iterative scheme for refining parameters. During iterations, we discard the image when the residual of transformation is larger than the triple of root-meansquare error (RMSE).

$$
\begin{aligned}
& \kappa_{i j}=\operatorname{artan} \frac{s_{i j} \cdot X_{r e l}^{i j}-s_{i j} \cdot X_{r e l}^{i}}{s_{i j} \cdot Y_{r e l}^{i j}-s_{i j} \cdot Y_{r e l}^{i}}-\operatorname{artan} \frac{X_{G P S}^{i j}-X_{G P S}^{i}}{Y_{G P S}^{i j}-Y_{G P S}^{i}} \\
& R_{\kappa_{i j}}=\left[\begin{array}{ccc}
\cos \kappa_{i j} & -\sin \kappa_{i j} & 0 \\
\sin \kappa_{i j} & \cos \kappa_{i j} & 0 \\
0 & 0 & 1
\end{array}\right] \\
& {\left[\begin{array}{lll}
X_{\kappa}^{i j} & Y_{\kappa}^{i j} & Z_{\kappa}^{i j}
\end{array}\right]^{T}=R_{\kappa_{i j}}\left[\begin{array}{lll}
X_{r e l}^{i j} & Y_{r e l}^{i j} & Z_{r e l}^{i j}
\end{array}\right]^{T}} \\
& {\left[\begin{array}{lll}
X_{\kappa}^{i} & Y_{\kappa}^{i} & Z_{\kappa}^{i}
\end{array}\right]^{T}=R_{\kappa_{i j}}\left[\begin{array}{lll}
X_{r e l}^{i} & Y_{r e l}^{i} & Z_{r e l}^{i}
\end{array}\right]^{T}} \\
& \omega_{i j}=\operatorname{artan} \frac{Y_{\kappa}^{i j}-Y_{\kappa}^{i}}{Z_{\kappa}^{i j}-Z_{\kappa}^{i}}-\operatorname{artan} \frac{Y_{G P S}^{i j}-Y_{G P S}^{i}}{Z_{G P S}^{i j}-Z_{G P S}^{i}} \\
& R_{\omega_{i j}}=\left[\begin{array}{ccc}
1 & 0 & 0 \\
0 & \cos \omega_{i j} & -\sin \omega_{i j} \\
0 & \sin \omega_{i j} & \cos \omega_{i j}
\end{array}\right] \\
& {\left[\begin{array}{lll}
X_{\kappa \omega}^{i j} & Y_{\kappa \omega}^{i j} & Z_{\kappa \omega}^{i j}
\end{array}\right]^{T}=R_{\omega_{i j}}\left[\begin{array}{lll}
X_{\kappa}^{i j} & Y_{\kappa}^{i j} & Z_{\kappa}^{i j}
\end{array}\right]^{T}} \\
& {\left[\begin{array}{lll}
X_{\kappa \omega}^{i} & Y_{\kappa \omega}^{i} & Z_{\kappa \omega}^{i}
\end{array}\right]^{T}=R_{\omega_{i j}}\left[\begin{array}{lll}
X_{\kappa}^{i} & Y_{\kappa}^{i} & Z_{\kappa}^{i}
\end{array}\right]^{T}} \\
& \varphi_{i j}=\operatorname{artan} \frac{X_{\kappa \omega}^{i j}-X_{\kappa \omega}^{i}}{Z_{\kappa \omega}^{i j}-Z_{\kappa \omega}^{i}}-\operatorname{artan} \frac{X_{G P S}^{i j}-X_{G P S}^{i}}{Z_{G P S}^{i j}-Z_{G P S}^{i}}
\end{aligned}
$$

where $\kappa_{i j}, \omega_{i j}, \varphi_{i j}=$ rotation angles computed from neighbor image $j$ in group $i$

After transforming the position and orientation of images from the free network coordinate system into the GPS coordinate system, each image contains several sets of exterior elements because it can possibly belong to different groups and can calculate a set of exterior elements of the image from one group. Thus, the parameters should be optimized before bundle adjustment. In this paper, each exterior element is processed individually with weighted least square estimation. Elements may be yielded from image pair with small overlap caused by long distance between the images, thereby affecting the preciseness of the relative orientation. Nevertheless, the long distance to the reference image is beneficial for space coordinate transformation. The initial weight of the exterior element from any set is determined as 1 because of data particularity. In this step, we implement the iterative scheme. Weights are determined using Equation (4) during the iterations.

$$
w(t, \sigma)=\left\{\begin{array}{cc}
0, & |t| \geq 3 \sigma \\
t^{2} /(3 \sigma)^{2}, & \sigma<|t|<3 \sigma \\
1, & |t| \leq \sigma
\end{array}\right.
$$

$$
\text { where } \quad \begin{aligned}
w & =\text { weight } \\
t & =\text { residual } \\
\sigma & =\text { root-mean-square error }
\end{aligned}
$$

Notably, if the final RMSE exceeds the threshold when determining the optimal initial exterior elements of an image for bundle adjustment, then the image will be discarded because of limited overlap with other images. The eliminated images are not considered in the final bundle adjustment. 
The initial exterior elements of images for bundle adjustment are obtained, and the coarse image network is established. The initial parameters obtained are reliable because GPS information is employed during network generation. Then, selfcalibration bundle adjustment with GPS information is implemented to refine the image network. As the GPS coordinates are in low precision, the weight of these coordinates should not be high.

\section{EXPERIMENTS}

\subsection{Test fields}

The proposed method for establishing an image network without accurate camera intrinsic parameters can provide a reliable image network with low-precision GPS information. To verify the efficiency of this method, we perform experiments on three test fields, designated as cases I, II, and IIII. Case I is the experiment performed in the test field in Huishan District in Jining, Shandong Province, China, an area near the lake. Case II is the test field in Luogang District in Guangzhou, Guangdong Province, China. This region contains mountain terrains, with a height difference reaching $350 \mathrm{~m}$. Case III is the experiment in the test field in Brazos County, Texas, USA. Other parameters of the experiments are shown in Table 1. The accuracy of GPS coordinates is about $10 \mathrm{~m}$.

\begin{tabular}{|c|c|c|c|}
\hline & Case I & Case II & Case III \\
\hline $\begin{array}{c}\text { Relative flight } \\
\text { altitude }\end{array}$ & $180 \mathrm{~m}$ & $950 \mathrm{~m}$ & $100 \mathrm{~m}$ \\
$\begin{array}{c}\text { Image amount } \\
\text { Camera model }\end{array}$ & 507 & 968 & 199 \\
Fonyl length & $16 \mathrm{~mm}$ & Canon 5DIII & Nikon J3 \\
Image format & $6000 \times 4000$ & $5760 \times 3840$ & $4608 \times 3072$ \\
size & $3.9 \mu \mathrm{mm}$ & $6.25 \mu \mathrm{m}$ & $2.86 \mu \mathrm{m}$ \\
Image pixel size & $4.4 \mathrm{~cm}$ & $11.9 \mathrm{~cm}$ & $2.9 \mathrm{~cm}$ \\
GSD & \multicolumn{2}{|l}{} \\
\hline
\end{tabular}

Table 1. Parameters of the experiments.

\subsection{Analysis and discussion of results}

We use thumbnail photos aligned according to a tracking map and rotated according to the yielded yaw angle of each image to approximately present the performance of the image network established using the proposed method. Yaw angles are calculated before the bundle adjustment. After the initial image network is generated, the overlapping areas among the rotated thumbnail photos should be presented similarly when the pitch and roll angles are not very large in UAV photogrammetry. We also present the point clouds generated before and after the bundle adjustment to prove that the established initial image network can guarantee the convergence of self-calibration bundle adjustment, and this initial network can be refined after self-calibration bundle adjustment with low-precision GPS coordinates as constraints. Since we do not have the control points in these fields, we just take the experiments to verify that the initial exterior elements of images calculated in our paper is valid and can guarantee high precision measurements when there are control points. The following sections present the results.
3.2.1 Case I: The obtained UAV images conform to convention photogrammetry, and the survey is conducted at the region without great topographic relief. The lake covers a large area of images at the left of Figure 3, which complicates the reconstruction of position and orientation because it is difficult to determine matching points from the region of water. As shown in Figure 3, the thumbnails are rotated according to the calculated yaw angles in the GPS coordinate system. The details in Figure 3 are shown in Figure 3. Figure 3(a), (b), and (c) indicate that the image network of this test field is recovered, and the orientation of the overlapping areas from different images are the same. Images photographed beside the lake are well reconstructed [Figure 3(a)] because we adopt the restriction that translation in $\mathrm{Z}$ direction should not be larger than the values in the two other directions at the same time. The point clouds generated before and after bundle adjustment are presented in Figure 4. Blue points denote the lower point in height, and red points are the higher ones. The coarse terrain is reconstructed before the bundle adjustment. The quality of the point cloud is improved after self-calibration bundle adjustment, thereby refining the image network.

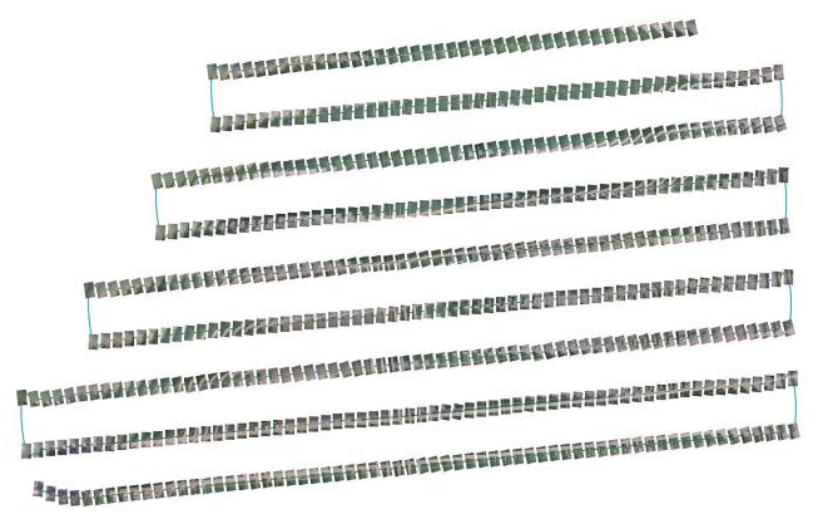

Figure 2 Tracking map with thumbnail photos rotated.



(a)

(b)

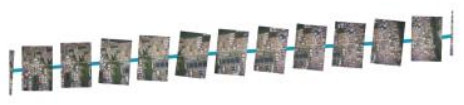

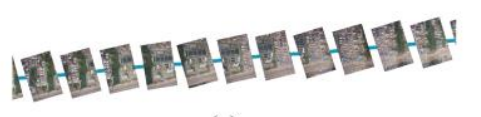

(c)

Figure 3. Parts of tracking map with thumbnail photos rotated. (a), (b) and (c) shows the details in track map with images rotated according to yaw angles calculated when initial image network generation in Case I. 


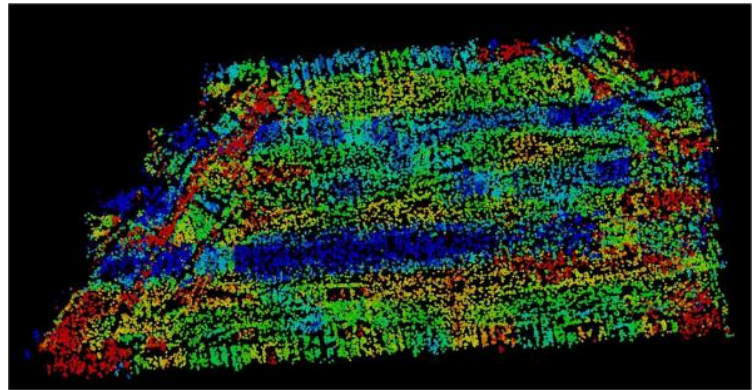

(a)



(b)

Figure 4. Point clouds. (a) and (b) show the point clouds before and after the self-calibration bundle adjustment with GPS information of Case I, respectively.

3.2.2 Case II: The obtained UAV images do not satisfy convention photogrammetry because some input images are captured under varied fly direction of UAV. Generally, these images cannot meet the requirements of the measurement. Thus, we should eliminate these images when establishing an image network. Figure 5 shows thumbnails rotated according to the calculated yaw angles in the GPS coordinate system. The discarded images are presented without rotation because their yaw angles cannot be calculated. For example, the images photographed when the machine took off and the images captured as the machine changes its direction are presented in horizontal. The details in Figure 5 are shown in Figure 6(a), (b), and (c). In Figure 6(a), images in the middle strip containing the suburban area are rotated to the right direction, and the road is in the same direction. In Figure 6(c), images near the corners of strips containing the mountain are recovered. Figure 6(d) shows the corner images in strips. The images possess few overlapping areas, and rotation angles are not obtained. Figure 7 shows the generated point clouds of this test field before and after the bundle adjustment. After the adjustment, the shape of mountains in the middle of the field can be refined. The left corner shows the suburban area with residents; this area is at a low height for convenient traveling.

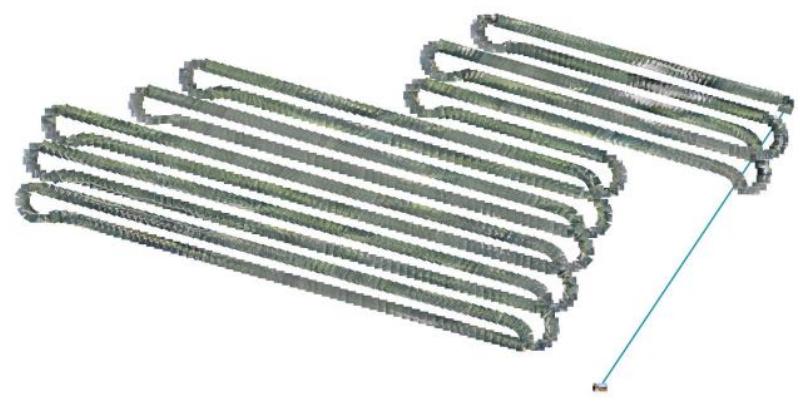

Figure 5 Tracking map with thumbnail photos rotated.

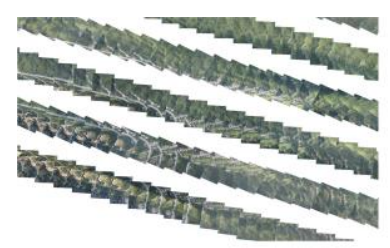

(a)

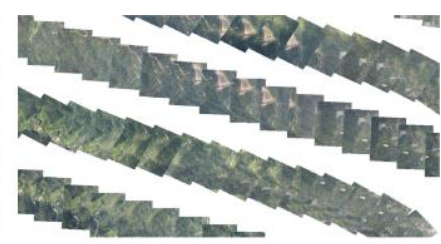

(b)

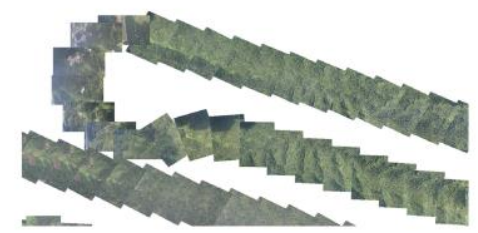

(c)

Figure 6. Parts of tracking map with thumbnail photos rotated. (a), (b) and (c) shows the details in track map with images rotated according to yaw angles calculated when initial image network generation in Case II.

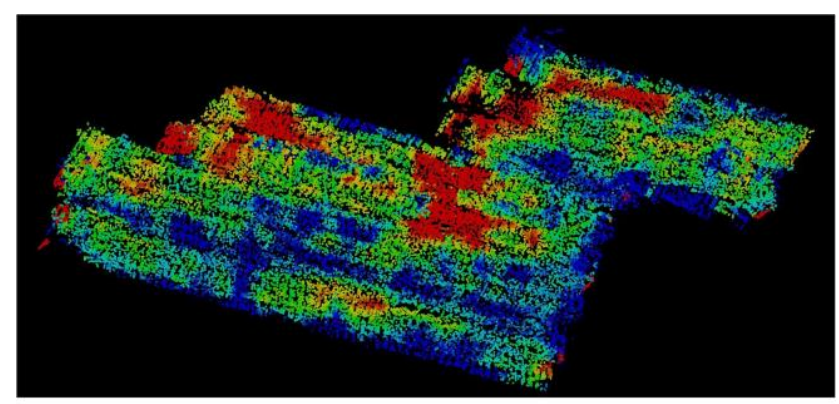

(a)

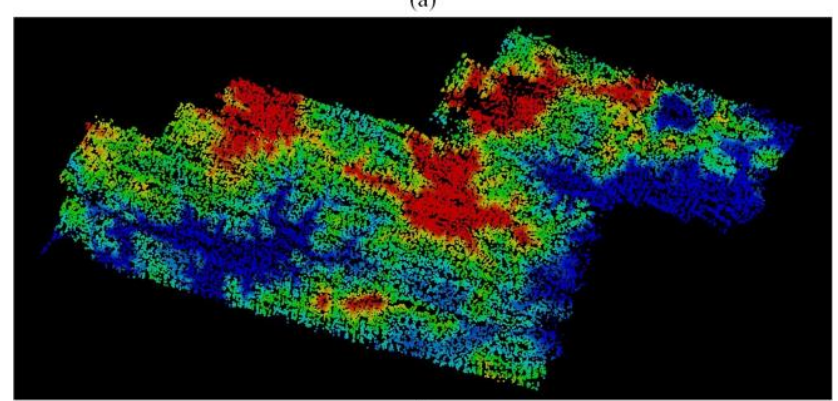

(b)

Figure 7. Point clouds. (a) and (b) show the point clouds before and after the self-calibration bundle adjustment with GPS information in Case II, respectively.

3.2.3 Case III: The UAV images are obtained arbitrarily. In contrast to those of case II, the images are still oriented to the ground when the machine changes its direction. As images are mainly rotated around $\mathrm{Z}$ axis and the overlapping areas are sufficient to ensure relative orientation between the images, thus the overlapped parts are retained. Figure 8 shows the tracking map with thumbnails rotated according to the calculated yaw angles in the GPS coordinate system after initial image network generation with all images are retained. Moreover, Figure 9 (a), (b), and (c) show details in Figure 8. The overlapping areas in different images are rotated to approximately the same direction. Thus, the initial image network is established. Figure 10 shows the point clouds generated before and after the bundle adjustment. Similarly, bundle adjustment improves the quality 
of point cloud. Hence, the established initial image network is valid and can be ameliorated by bundle adjustment.

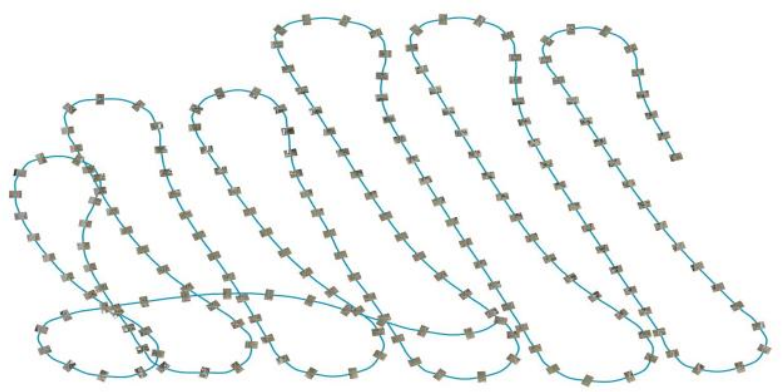

Figure 8 Tracking map with thumbnail photos rotated.

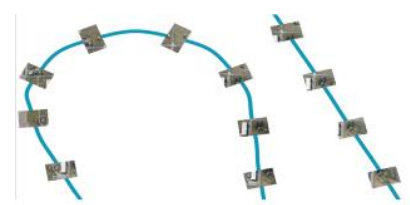

(a)



(b)



(c)

Figure 9. Parts of tracking map with thumbnail photos rotated. (a), (b) and (c) shows the details in track map with images rotated according to yaw angles calculated when initial image network generation in Case II.

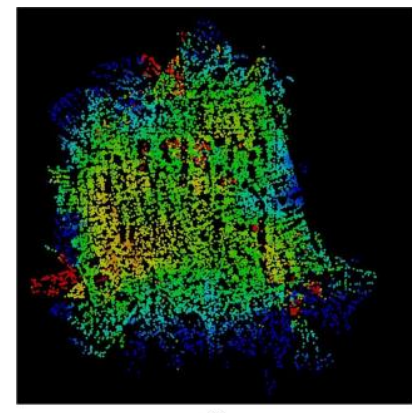

(a)

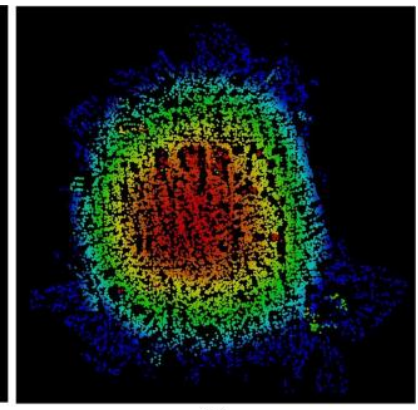

(b)
Figure 10. Point clouds. (a) and (b) show the point clouds before and after self-calibration bundle adjustment with GPS information of Case III.

\section{CONCLUSION}

Considering the limited carrying capacity of UAVs, researchers generally use light-weight non-metric digital cameras for these devices. However, some non-metric digital cameras used for measurement are not calibrated; as such, camera model parameters are inaccurate, thereby complicating image network generation. In this paper, our main contribution is to introduce the use of low-precision GPS information for improving the robustness of image network generation. When using GPS information, it avoid matching all images with one another to obtain the overlaps among images, and the efficiency of data procession will be greatly improved in theory. We divide images into groups and generate a free network for each group. The low-precision GPS coordinates of the images are then used to transform the exterior elements of images from the free network coordinate system into the GPS coordinate system to establish the initial image network. This initial network contributes to the convergence of the self-calibration bundle adjustment and can be refined after the adjustment.

In the experiments, UAV images with regular and non-regular stripes are employed to validate the proposed method. The method can be used for effective image network generation. And it confirms that the initial parameters for bundle adjustment computed by our method are valid.

Meanwhile, following problems remain to be solved in future works.

i. When determining the optimal set of exterior elements of each image as initial parameters for bundle adjustment, we simplify the process by working on each element individually with weighted least square estimation. However, this process destroys the relations among the elements of images. Thus, a global optimal algorithm to composite the data must be developed.

ii. We designate each image as the reference and then grouped with its neighbor images. This process ignores the characteristic that most UAV images are captured in sequence, thereby decreasing efficiency because of redundant operations. This limitation must be addressed to improve efficiency of data processing.

iii. We will conduct the test fields with control points to evaluate the precision achieved our method, and compare it with other commercial softwares.

\section{ACKNOWLEDGEMENTS}

This work was supported in part by the National Natural Science Foundation of China under Grants 41201482, the Research Funds of the Key Laboratory of Mapping from Space, National Administration of Surveying, Mapping and Geoinformation with project number K201405, the Fundamental Research Funds for the Central Universities with project number 2014213020201. The authors would like to thank the anonymous reviewers, members of the editorial team, He Huang and Mongxiao Song from Wuhan University, China, and Kai Liu for their comments and contributions, which have significantly improved this paper.

\section{REFERENCES}

Agarwal, S., Furukawa, Y. and Snavely, N., et al., 2011. Building rome in a day. Communications of the ACM, 54(10), pp. 105-112.

Bemis, S. P., Micklethwaite, S. and Turner, D., et al., 2014. Ground-based and UAV-based photogrammetry: a multi-scale, high-resolution mapping tool for structural geology and paleoseismology. Journal of Structural Geology, 69, pp. 163178.

Fischler, M. A. and Bolles, R. C., 1981. Random sample consensus: a paradigm for model fitting with applications to image analysis and automated cartography. Communications of the ACM, 24(6), pp. 381-395. 
Gonçalves, J. A. and Henriques, R., 2015. UAV photogrammetry for topographic monitoring of coastal areas. ISPRS Journal of Photogrammetry and Remote Sensing, 104, pp. 101-111.

Huang, S., Zhang, Z. and Ke, T., et al., 2015. Scanning photogrammetry for measuring large targets in close range. Remote Sensing, 7(8), pp. 10042-10077.

Hui, Y., Xhiping, C. and Shanjia, X., et al., 1998. An unmanned air vehicle (UAV) GPS location \& navigation system. In: International Conference on Microwave and Millimeter Wave Technology Proceedings, Beijing, China, pp. 472-475.

Jiang, N., Cui, Z. and Tan, P., 2013. A global linear method for camera pose registration. In IEEE International Conference on Computer Vision, pp. 481-488.

Lowe, D. G., 2004. Distinctive image features from scaleinvariant keypoints. International Journal of Computer Vision, 60(2), pp. $91-110$.

Luhmann, T., 2010. Close range photogrammetry for industrial applications. ISPRS Journal of Photogrammetry and Remote Sensing, 65(6), pp. 558-569.

Mammarella, M., Campa, G. and Napolitano, M. R., et al., 2008. Machine vision/GPS integration using EKF for the UAV aerial refueling problem. IEEE Transactions on Systems, Man, and Cybernetics, Part C (Applications and Reviews), 38(6), pp. 791-801.

Nemra, A. and Aouf, N., 2010. Robust INS/GPS sensor fusion for UAV localization using SDRE nonlinear filtering. IEEE Sensors Journal, 10(4), pp. 789-798.

Schaffalitzky, F. and Zisserman, A., 2002. Multi-view matching for unordered image sets, or "how do I organize my holiday snaps?". In: Lecture Notes in Computer Science, Berlin, Germany, pp. 414-431.

Slama, C., Theurer, C. and Hendrikson, S. W., 1980. Manual of photogrammetry. American Society of Photogrammetry, Falls Church, VA.

Stewenius, H., Engels, C. and Nister, D., 2006. Recent developments on direct relative orientation. ISPRS Journal of Photogrammetry and Remote Sensing, 60(4), pp. 284-294.

Strecha, C., von Hansen, W. and Van Gool, L., et al., 2008. On benchmarking camera calibration and multi-view stereo for high resolution imagery. In IEEE Computer Society Conference on Computer Vision and Pattern Recognition, New York, USA, pp. 2838-2845.

Uysal, M., Toprak, A. S. and Polat, N., 2015. Dem generation with UAV photogrammetry and accuracy analysis in sahitler hill. Measurement, 73, pp. 539-543.

Wang, Z., 2007. Principle of photogrammetry. Wuhan Unviversity Press, Wuhan.

Westoby, M. J., Brasington, J. and Glasser, N. F., et al., 2012. 'structure-from-motion' photogrammetry: a low-cost, effective tool for geoscience applications. Geomorphology, 179, pp. 300-314.

Wilson, K. and Snavely, N., 2014. Robust global translations with 1DSFM. In Lecture Notes in Computer Science, CHAM, pp. 61-75.

Wu, C., 2014. Critical configurations for radial distortion selfcalibration. In: IEEE Conference on Computer Vision and Pattern Recognition, Columbus, USA, pp. 25-32. 